\title{
Different magnitude of resistance to non-depolarizing muscle relaxants in dexamethasone-treated rat diaphragm associated with altered acetylcholine receptor expression
}

\author{
D. Chen, M.R. Yang, L.N. Huang, Y.W. Qiu and S.T. Li \\ Department of Anesthesiology, First People's Hospital, School of Medicine, \\ Shanghai Jiaotong University, Shanghai, China \\ Corresponding author: S.T. Li \\ E-mail: zhngweilv@126.com
}

Genet. Mol. Res. 13 (3): 5892-5900 (2014)

Received March 28, 2013

Accepted February 11, 2014

Published August 7, 2014

DOI http://dx.doi.org/10.4238/2014.August.7.4

\begin{abstract}
The aim of this study was to investigate the influence of chronic dexamethasone (Dex) administration on rat diaphragm sensitivity to non-depolarizing muscle relaxants (NDMRs) and muscular nicotinic acetylcholine receptor (nAChR) expression, which may help direct future administration of NDMRs. Adult male SpragueDawley rats were randomized to receive a daily intraperitoneal injection of Dex $(600 \mu \mathrm{g} / \mathrm{kg}$ body mass) or an equivalent volume of saline $(\mathrm{N}=$ 20 in each group) for 14 days. We evaluated isometric twitch tensions of nerve-hemidiaphragm preparations elicited by indirect supramaximal stimulation at $0.1 \mathrm{~Hz}$. Real-time quantitative PCR was performed to determine the mRNA expression of two nAChR subunits ( $\varepsilon$-subunit and $\gamma$-subunit) in the diaphragm. Dex administration markedly $(\mathrm{P}<0.01)$ increased the $50 \%$ twitch depression $\left(\mathrm{IC}_{50}\right)$ of the three NDMRs. The $\mathrm{IC}_{50}$ ratio, which standardized the magnitudes of the resistance, was the
\end{abstract}


largest for atracurium, with the second largest for vecuronium and the smallest for rocuronium $(\mathrm{P}<0.01)$. The $\varepsilon$ - and $\gamma$-subunit mRNAs were both upregulated with an increased $\gamma / \varepsilon$ ratio in rats exposed to Dex. The results indicated that chronic Dex administration induces hyposensitivity to NDMRs, the degree of which depends on the kind of neuromuscular blocker, and is associated with increased nAChR expression.

Key words: Glucocorticoids; Neuromuscular blocking agents; Acetylcholine receptor; Hemidiaphragm

\section{INTRODUCTION}

Muscle atrophy and resistance to the actions of non-depolarizing muscle relaxants (NDMRs) are commonly associated with chronic glucocorticoid treatment.

Soltész et al. (2008, 2009) found a shorter duration of atracurium (ATR)-induced neuromuscular block in patients receiving long-term prednisolone medication and later confirmed that this was also valid for rocuronium (ROC). Parr et al. (1991) observed that patients on long-term betamethasone treatment required $75 \%$ more vecuronium (VEC). In experimental settings, chronic dexamethasone (Dex) treatment antagonized the neuromuscular blocking effect of d-tubocurarine manifested by increasing the $\mathrm{LD}_{50}$ of d-tubocurarine and shortening the duration of neuromuscular block (Arts and Oosterhuis, 1977; Leeuwin et al., 1981). Thus, chronic glucocorticoid-induced hyposensitivity to NDMRs does not seem to be limited to a certain group (benzylisoquinoline or aminosteroidal class). Due to different methods, it is not easy to make a direct comparison of the above data, and whether the magnitude of resistance differs between drugs is not clear.

Mechanisms of resistance to NDMRs in chronic glucocorticoid treatment have not been investigated extensively. The beneficial effect of corticosteroids in deficient neuromuscular transmission (e.g., in myasthenia gravis) is due not only to an effect on the immune system but also to a direct effect on muscle (Kaplan et al., 1990). Braun et al. (1993) observed that long-term treatment with glucocorticoids (hydrocortisone, prednisolone or Dex) decreased degradation and increased synthesis of junctional nicotinic acetylcholine receptor (nAChRs). Kaplan et al. (1990) found that Dex significantly increases total surface nAChRs in cultured human muscle exposed to myasthenia gravis serum. Furthermore, with the single channel patch-clamp technique, Maestrone et al. (1995) proved that Dex-upregulated nAChRs were functional and that their electrophysiological parameters were similar to those found in control myotubes. Muscle nAChRs include two subtypes: the adult mature form ( $\varepsilon$-AChR) composed of $\alpha_{2} \beta \delta \varepsilon$ subunits and the fetal immature form ( $\gamma$-AChR) containing $\alpha_{2} \beta \delta \gamma$ subunits. $\gamma$-AChR is initially expressed in fetal muscle, and then replaced by $\varepsilon$-AChR when muscles are innervated. However, in some pathologic conditions (e.g., denervation), $\gamma$-AChR is re-expressed throughout the muscle membrane (Martyn et al., 2009; Fagerlund and Eriksson, 2009). The expression of $\gamma$-AChR has clinical significance, because the two subtypes have different electrophysiological and drug-binding properties (Martyn et al., 1992). Upregulation and spreading of nAChRs, especially the immature form, can induce resistance to NDMRs, as documented in burns, denervation and immobilization (Martyn et al., 1992; Martyn and Richtsfeld, 2006; Jeevendra et al., 2006). Thus, Dex-induced upregulation of functional nAChRs can lead to resistance to NMBRs (Maestrone et al., 1995). 
Our previous research suggested that the magnitude of denervation-induced resistance to NDMRs depends on the kind of neuromuscular blocker and is related to the different potencies of individual NDMRs on nAChR subunits (Wang et al., 2010). The hypothesis of this study was that chronic Dex treatment would cause different magnitudes of resistance to different NDMRs related to altered expression of nAChRs.

We have found resistance to ROC in Dex-treated rats demonstrated by a shift of the ROC concentration-twitch tension curves to the right (Chen et al., 2014). The aim of this study was to determine the differences in Dex-induced hyposensitivity to three NDMRs. Additionally, we investigated nAChRs subunit mRNA expression.

\section{MATERIAL AND METHODS}

\section{Animals and muscle preparation}

The study was approved by the Animal Care and Use Committee of the School of Medicine, Shanghai Jiaotong University and was performed in compliance with the WHO International Guiding Principles for Animal Research.

Adult male Sprague-Dawley rats (180-220 g) were randomly allocated into two groups (20 animals each). The Dex group received daily intraperitoneally injections of Dex (600 $\mu \mathrm{g} /$ $\mathrm{kg}$ body mass), a dose previously shown to cause muscle atrophy (Ma et al., 2003; Yamamoto et al., 2010) and to induce diaphragm hyposensitivity to ROC (Chen et al., 2014) prepared in isotonic vehicle $(0.9 \% \mathrm{NaCl})$, and the saline group received an equivalent volume of saline $(0.9 \% \mathrm{NaCl})$, both for 14 days. All injections were performed at approximately the same time of day. Animal mass was recorded daily and Dex doses were adjusted to changes in body mass. The rats in both groups had free access to food, and water ad libitum. At $24 \mathrm{~h}$ after the last Dex dose, the rats were anesthetized with pentobarbital $(60 \mathrm{mg} / \mathrm{kg}$ intraperitoneally). The left hemidiaphragm with attached phrenic nerve, central tendon, and rib cage intact were rapidly moved.

\section{Measurement of isometric twitch contractions}

Strips of diaphragm (8-10 mm wide) with the phrenic nerve attached, which had been cut parallel to the muscle fibers, were dissected. Each isolated strip was mounted vertically in a tissue chamber, fixed at the rib cage, inferiorly positioned, and suspended at the central tendon from a force displacement transducer by using a 3-0 silk suture (ALC-M System for Isolated Tissue-Organ Research, $40 \mathrm{~mL}$ in volume). The chamber was filled with Krebs solution maintained at $37^{\circ} \mathrm{C}$ and bubbled with $95 \% \mathrm{O}_{2} 5 \% \mathrm{CO}_{2}$. The composition of the Krebs solution was: $137 \mathrm{mM} \mathrm{NaCl}, 4 \mathrm{mM} \mathrm{KCl}, 2 \mathrm{mM} \mathrm{CaCl}, 1 \mathrm{mM} \mathrm{MgCl}, 1 \mathrm{mM} \mathrm{KH}_{2} \mathrm{PO}_{3}, 12 \mathrm{mM}$ $\mathrm{NaHCO}_{3}$ and 6.5 glucose, while $\mathrm{pH}$ was $7.40 \pm 0.05$ during bubbling.

Isometric twitch tension was elicited by indirect (phrenic nerve) or direct (muscle) supramaximal constant-voltage stimulation at $0.1 \mathrm{~Hz}$ (for 0.05 and for $0.2 \mathrm{~ms}$ in the cases of indirect and direct stimulation, respectively) by using a stimulator and a constant-voltage unit with an optimal preload (1.0-2.0 g) at which skeletal muscle can generate the greatest force. The phrenic nerve was positioned on wire bipolar silver electrodes for indirect stimulation. For direct stimulation, the diaphragm was placed in between two large silver electrodes, and pretreated with a sufficient concentration $(5 \mu \mathrm{M})$ of d-tubocurarine to completely abolish neuromuscular transmission. The twitch tension was recorded via the force transducer on a re- 
corder (ALC-MPA 2000m, Acquisition and Analysis System for Life Science Research, Alcott Biotech, Shanghai, China).

After the elicited twitch tension had been stabilized for at least 15 min, single-twitch tension (averaged in groups of five) was determined. A drug was then applied to the preparation. The drug concentrations were determined by adding freshly prepared solutions with calibrated micropipettes to $40 \mathrm{~mL}$ of modified Krebs solution in the tissue chamber. After stabilization of the drug effect for at least $10 \mathrm{~min}$, single-twitch tension was again determined. Data were accepted only when twitch tension returned to $95-105 \%$ of the initial value by rinsing the diaphragm preparation with Krebs solution in each study. ROC bromide was obtained from N.V. Organon (Oss, The Netherlands), and all other drugs were purchased from Sigma (St. Louis, MO, USA).

Following the experiment, the muscle was weighed, and the cross-sectional area (CSA) was estimated based on the following formula: muscle weight $(\mathrm{g}) /\left[\mathrm{L}_{0}(\mathrm{~cm}) \times 1.056(\mathrm{~g} /\right.$ $\left.\mathrm{cm}^{3}\right)$ ] (assuming muscle density $\left.=1.056 \mathrm{~g} / \mathrm{cm}^{3}\right)($ Van Balkom et al., 1997). Indirect and direct twitch tensions were then normalized to the CSA of the muscle segments.

\section{Real-time reverse transcription polymerase chain reaction (RT-PCR)}

Quantification of mRNA coding for nAChR subunit genes ( $\varepsilon$ - and $\gamma$-subunits) was done using the real-time RT-PCR technique (see review by Garcia and Ma, 2005). The procedure may be summarized as follows: total RNA was extracted from diaphragms using Trizol reagent (Invitrogen, Cergy Pontoise, France) and RNeasy Mini lit (Qiagen, Valencia, CA, USA) according to manufacturer instructions. After extraction, RNA was stored at $-20^{\circ} \mathrm{C}$. The concentration and purity of the RNA was determined spectrophotometrically by measuring the absorbance at 260 and $280 \mathrm{~nm}$ with a GeneQuant RNA/DNA Calculator (Amersham Pharmacia Biotech, Orsay, France): an $\mathrm{A}_{260} / \mathrm{A}_{280}$ ratio in the range 1.7 to 2.0 was considered acceptable. The structural integrity of the RNA was verified by electrophoresis on $2 \%$ agarose gels followed by ethidium bromide staining to visualize $18 \mathrm{~S}$ and $28 \mathrm{~S}$ ribosomal RNA. All primers used in this study were designed by Sangon Biotech Co. Ltd. (Shanghai, China). The primer sequences were as follows: $\varepsilon$-subunit (forward: 5'-CCAAGGCAAGAATGAA-3', reverse: 5'-TAGGTCTGGGAACGAA-3'); $\gamma$-subunit (forward: 5'-CTGGGACCCGAAAGAC-3', reverse: 5'-TCGCTGGTGCTGTAAGT3'); $\beta$-actin (forward: 5'-TGG AAT CCT GTG GCA TCC ATG AAA C-3', reverse: 5'-TAA AAC GCA GCT CAG TAA CAG TCC G-3'). Since our preliminary experiment confirmed that the expression level of $\beta$-actin in rat diaphragm was not modified after Dex treatment, the levels of $\beta$-actin mRNA were used to control for inter-sample variations in the amount of RNA used for the RT-PCRs. RT-PCR for detection of $\varepsilon$ - and $\gamma$-subunits and $\beta$-actin mRNAs was performed in individual tubes using $1 \mu \mathrm{g}$ total RNA and the SuperScript One-Step RT-PCR kit (Life Technologies, Grand Island, NY, USA) with the following conditions. The reverse transcription (RT) reaction was performed at $50^{\circ} \mathrm{C}$ for $30 \mathrm{~min}$. After an initial denaturation step at $94^{\circ} \mathrm{C}$ for $5 \mathrm{~min}$, 35 cycles $\left(45 \mathrm{~s}\right.$ at $94^{\circ} \mathrm{C}, 45 \mathrm{~s}$ at $55^{\circ} \mathrm{C}$ and $1 \mathrm{~min}$ at $72^{\circ} \mathrm{C}$ ) of PCR were run to obtain the $\varepsilon, \gamma$ and $\beta$-actin products. All measurements were performed in triplicate. For each sample, the threshold cycle $(\mathrm{Ct})$ was calculated on the basis of the cycle at which the fluorescence increased above a threshold level. The $\Delta \mathrm{Ct}$ values were calculated in every sample for the target gene as follows: $\mathrm{Ct}$ (target gene)-Ct (internal control gene), with $\beta$-actin as internal control gene. The relative expression level for one target gene ( $\Delta \Delta \mathrm{Ct}$ ) was calculated by subtracting the mean $\Delta \mathrm{Ct}$ for the control group from the $\Delta \mathrm{Ct}$ of each sample in the treated groups. Finally, the relative expression value, normalized to an endogenous reference, was given by $2^{-\Delta \Delta C t}$. 


\section{Statistical analysis}

All statistical analyses were performed using the Social Sciences (SPSS)/PC + package (Chicago, IL, USA). Data are reported as means \pm standard deviation (SD) and compared using one-way or two-way repeated measures ANOVA with the post hoc Bonferroni test or repeated measures ANOVA was used for force-frequency and growth curve analysis. Twoway ANOVA with the post hoc Bonferroni analysis was used to detect differences of relative RNA levels between Dex and saline sides and $\mathrm{P}<0.05$ was accepted as statistically significant.

\section{RESULTS}

\section{Body weight}

There was no significant difference in the initial body weights of rats $(\mathrm{P}>0.05)$. At the end of the 14-day treatment period, body weights in the two experiment groups were significantly different from each other $(\mathrm{P}<0.01)$ (Table 1$)$.

\begin{tabular}{lcc}
\multicolumn{1}{c}{ Table 1. Experimental treatment on body weight. } & \\
\hline Group & & Body weight \\
\cline { 2 - 3 } & Initial (g) & Final (g) \\
\hline Dexamethasone & $197.55 \pm 2.688$ & $171.10 \pm 2.812^{*}$ \\
Saline & $194.70 \pm 2.163$ & $280.95 \pm 2.423$ \\
\hline
\end{tabular}

Values are reported as means $\pm \mathrm{SD}(\mathrm{N}=20$ in each group). Statistical analysis was carried out by one-way ANOVA with the post hoc Bonferroni test, ${ }^{\sharp} \mathrm{P}<0.05$ vs saline.

\section{Isometric twitch contractions}

There was no significant difference between groups with regard to diaphragm size (width and length), or in twitch tensions elicited by indirect or direct stimulation (7.8-17.4 g, $\mathrm{P}>0.05$, Figure $1 \mathrm{~A}$ and $\mathrm{B}$ ).
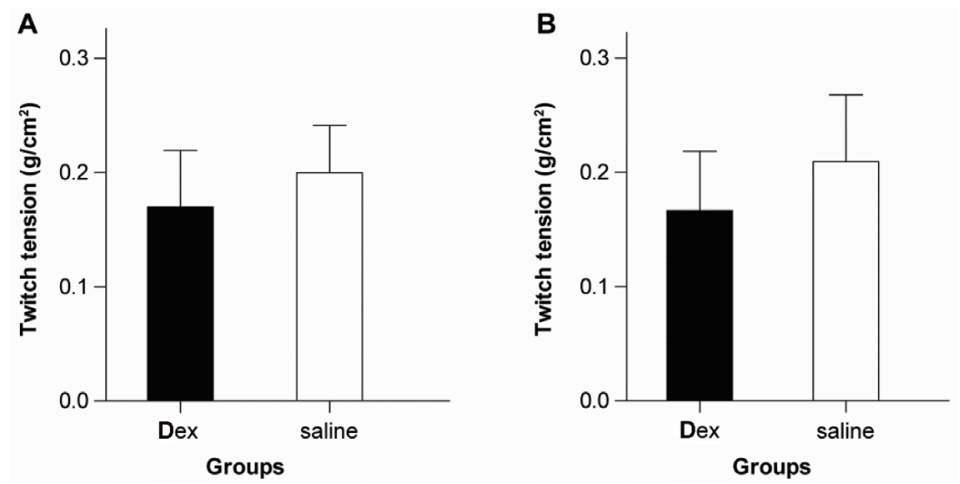

Figure 1. Single-twitch tension elicited by indirect (phrenic nerve; A) or direct (muscle; B) stimulation. Values are reported as means $\pm \mathrm{SD}(\mathrm{N}=20$ in each group). Statistical analysis was carried out by one-way ANOVA with the post hoc Bonferroni test. Dex = dexamethasone. 


\section{Potency of NDMRs}

After rinsing the diaphragms exposed to ROC with Krebs solution, the indirectly elicited twitch tension returned to $95-105 \%$ of the initial value in each study, which indicated that the diaphragm remained responsive.

In the saline group, ROC, VEC, and ATR reduced the indirectly elicited twitch tensions in a concentration-dependent way $(\mathrm{P}<0.05$; Figure $2 \mathrm{~A})$. The concentration-twitch tension curves of these neuromuscular blockers were significantly different $(\mathrm{P}<0.01$; Figure $2 \mathrm{~A}) . \mathrm{IC}_{50}$, which quantitatively indicates the position of the curve, was significantly higher for ROC, followed by ATR, and lower for VEC $(\mathrm{P}<0.01$; Table 2$)$. The slope was the largest in ROC, the second largest for ATR and the smallest for VEC $(\mathrm{P}<0.05$; Table 2$)$.
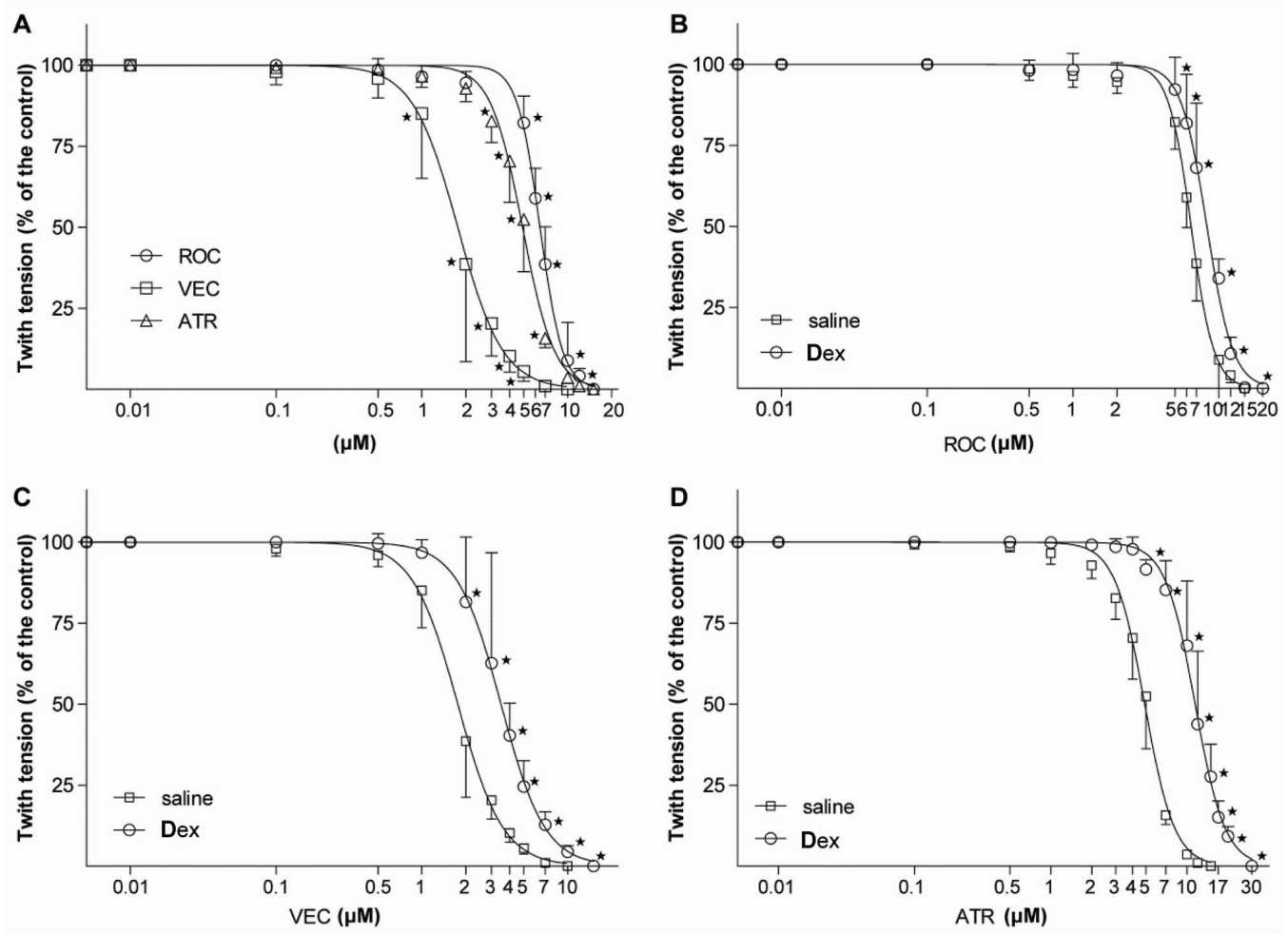

Figure 2. A. Concentration-twitch tension curves for rocuronium (ROC), vecuronium (VEC), and atracurium (ATR) in saline-treated rats. B. C. D. Concentration-twitch tension curves for ROC, VEC and ATR in rats from saline and dexamethasone (Dex) groups. Data are reported as means $\pm \mathrm{SD}(\mathrm{N}=20$ in each curve). Statistical analysis was carried out by two-way repeated measures ANOVA with the post hoc Bonferroni test. *P $<0.05 v s$ control value. For each NDMR, the curve was significantly shifted rightward in Dex from the saline group.

In the Dex group, ROC, VEC, and ATR reduced the magnitudes of indirectly elicited twitch tensions in a concentration-dependent way $(\mathrm{P}<0.05$; Figure $2 \mathrm{~B}, \mathrm{C}, \mathrm{D})$. For each NDMR, the concentration-twitch tension curve was significantly $(\mathrm{P}<0.01)$ shifted to the right compared with that in the saline group (Figure $2 \mathrm{~B}, \mathrm{C}, \mathrm{D}$ ). $\mathrm{The}_{\mathrm{IC}}$ for each NDMR in the Dex group was 
significantly ( $\mathrm{P}<0.01$; Table 2$)$ larger than that in the saline group. However, there was no significant difference between the slope in the Dex group and that in the saline group for each NDMR (Table 2), indicating no obvious drug interaction. The $\mathrm{IC}_{50}$ ratios, which standardize the magnitudes of the rightward shifts of the concentration-twitch tension curves, differed significantly ( $\mathrm{P}$ $<0.05$ ) with the largest for ATR, the second largest for VEC and the smallest for ROC (Table 2).

Table 2. $\mathrm{IC}_{50}$ values and slopes of the concentration-twitch tension curves of rocuronium (ROC), vecuronium (VEC), and atracurium (ATR) in dexamethasone (Dex) and saline groups.

\begin{tabular}{|c|c|c|c|}
\hline & ROC & VEC & ATR \\
\hline \multicolumn{4}{|l|}{ Saline } \\
\hline $\operatorname{LogIC}_{50}$ & $0.786 \pm 0.0384$ & $0.246 \pm 0.0108^{\S}$ & $0.690 \pm 0.0356^{\S \#}$ \\
\hline Slope & $-4.88 \pm 0.698$ & $-2.80 \pm 0.396^{8}$ & $-3.98 \pm 0.532^{\S \#}$ \\
\hline $\mathrm{IC}_{50}$ & $6.14 \pm 0.604$ & $1.76 \pm 0.182$ & $4.90 \pm 0.504$ \\
\hline \multicolumn{4}{|l|}{ Dex ${ }^{30}$} \\
\hline $\operatorname{LogIC}_{50}$ & $0.920 \pm 0.0618^{\prime \prime}$ & $0.542 \pm 0.0211^{\prime \prime}$ & $1.06 \pm 0.0409^{\natural}$ \\
\hline Slope ${ }^{30}$ & $-5.57 \pm 0.569$ & $-2.89 \pm 0.405$ & $-3.97 \pm 0.373$ \\
\hline $\mathrm{IC}_{8}$ & $8.39 \pm 1.14$ & $3.49 \pm 0.568$ & $11.5 \pm 1.18$ \\
\hline $\mathrm{IC}_{50}$ ratio & $1.27 \pm 0.0970$ & $1.93 \pm 0.170^{\S}$ & $2.38 \pm 0.105^{5^{\sharp}}$ \\
\hline
\end{tabular}

Data are reported as means $\pm \mathrm{SD}(\mathrm{N}=20$ in each group). Statistical analysis was carried out by one-way ANOVA with the post hoc Bonferroni test. ${ }^{\circledR} \mathrm{P}<0.05$ vs ROC, ${ }^{\sharp} \mathrm{P}<0.05$ vs $\mathrm{VEC},{ }^{\natural} \mathrm{P}<0.05$ vs saline.

\section{Quantification of mRNA for $\varepsilon-$ and $\gamma$ - subunit}

Real-time RT-PCR showed significant $(\mathrm{P}<0.05)$ increases in $\varepsilon$ - and $\gamma$-subunit mRNA expressions in Dex compared with the saline group (Figure $3 \mathrm{~A}$ and $\mathrm{B}$ ).
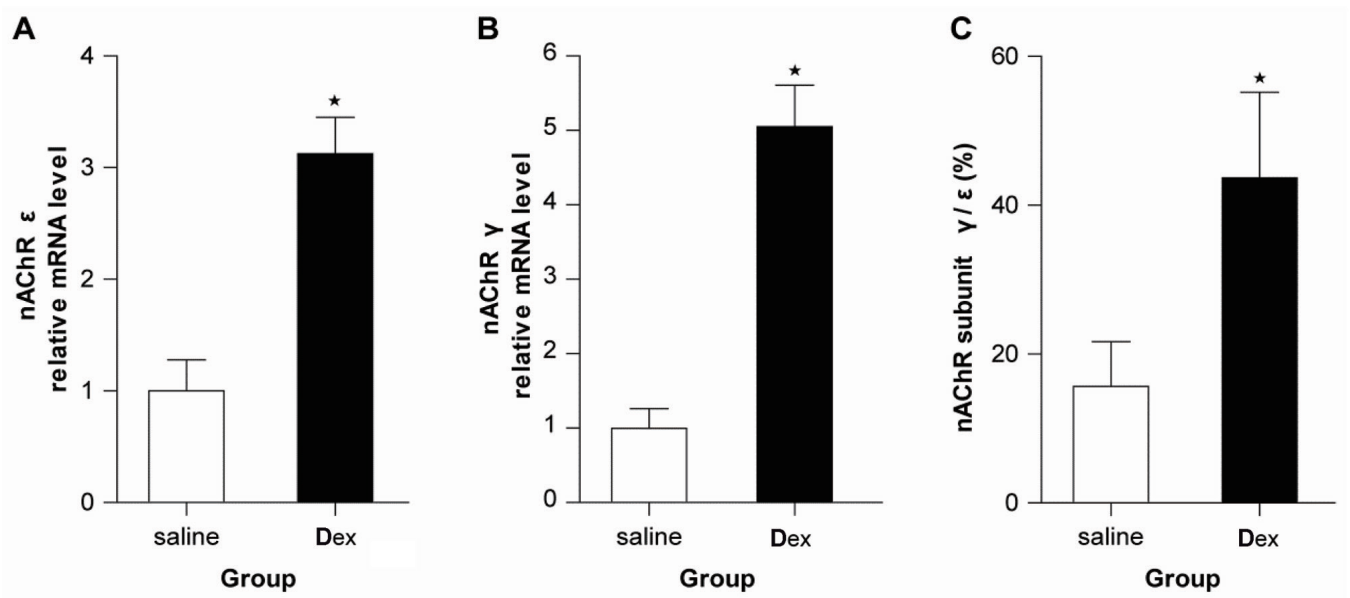

Figure 3. A. B. Relative mRNA expressions of $n A C h R ~ \varepsilon$ - and $\gamma$-subunits normalized to their respective $\beta$-actin expressions, and averaged with all samples in a group, with the control group as $100 \%$. C. nAChR subunit $\gamma / \varepsilon$ ratios in dexamethasone (Dex) and saline groups. Values are reported as means $\pm \mathrm{SD}(\mathrm{N}=20$ in each group). Statistical analysis was carried out by one- or two-way ANOVA with the post hoc Bonferroni test, $* \mathrm{P}<0.05$ vs saline group.

nAChR $\gamma / \varepsilon$ ratio was significantly $(\mathrm{P}<0.05)$ increased in the Dex group, indicating an increase in the percentage of immature nAChRs in diaphragm of the rats exposed to Dex (Figure 3C). 


\section{DISCUSSION}

In this study, daily medication with Dex over a period of 2 weeks differentially attenuated the neuromuscular blocking effect of ROC, VEC and ATR on indirectly elicited twitch tension of the rat diaphragm in vitro.

ROC and VEC are representative of neuromuscular blocking agents of the aminosteroidal class, while ATR is representative of the benzylisoquinolone class. Therefore, the reduced effect of NDMR does not seem to be limited to a certain molecular structure. These are in agreement of several previous investigations (Arts and Oosterhuis, 1977; Leeuwin et al., 1981; Parr et al., 1991; Soltesz et al., 2008, 2009). The $\mathrm{IC}_{50}$ ratios were different between the neuromuscular blockers, suggesting that the magnitude of Dex-induced attenuation depends on the kind of neuromuscular blocker.

Robinson et al. (1992) observed that betamethasone-induced resistance to neuromuscular blockage affected VEC and ATR to similar degrees. They used rat phrenic nervehemidiaphragm preparation bathed in a physiological solution with betamethasone. Thus, they studied the acute effect of betamethasone. In contrast, we investigated the long-term effect of Dex on actions of NDMRs. This may be the reason for the contradictory results.

Shin et al. (2000) measured the neuromuscular blocking effect of d-tubocurarine after 7-day prednisolone administration in the tibialis cranialis muscle in vivo and found a reduced $\mathrm{EC}_{50}$ of d-tubocurarine in steroid-treated rats. The discrepancy may come from several aspects. First, they used a non-fluorinated steroid (prednisolone), while we used a fluorinated one (Dex). Second, duration was prolonged from 7 to 14 days in our study. Third, they studied the in vivo effect of NDMR, which could not distinguish the impact on pharmacodynamics from that on pharmacokinetics (Shina, 1990).

We found increased expression of nAChRs, which would increase the amount of NDMR required to competitively block nAChRs, a possible mechanism for the resistance to NDMRs. We also observed an increased percentage of immature $\gamma$-subunit. According to our previous study on the actions of the NDMRs tested at $\gamma$ - and $\varepsilon$-nAChR expression in HEK293 cells, ATR was less potent at $\gamma$-nAChR than $\varepsilon$-nAChR, VEC was equipotent at both receptor subtypes, while ROC was more potent at $\gamma$-nAChR than $\varepsilon$-nAChR (Wang et al., 2010). Thus, the rank order of the magnitude of resistance to the three NDMRs in skeletal muscle with upregulation of immature nAChRs was ATR $>$ VEC $>$ ROC. In the present study, upregulation of nAChRs with increased $\gamma / \varepsilon$ ratio in the Dex-treated rat diaphragm can explain the different magnitudes: resistance to ATR was the greatest, which is consistent with the known lesser potency of ATR at $\gamma$-AChR compared with the potency at $\varepsilon$-AChR, and resistance to ROC was the least, which again is consistent with a greater potency at $\gamma$-AChR than at $\varepsilon$-AChR.

Knowing the different magnitudes of resistance to NDMRs following chronic steroid treatment is important for all anesthesiologists in their clinical practice. An unexpected decreased effect of the drug may contribute to poor muscle relaxation. Coughing and body movements can lead to intraoperative complications. Chronic Dex exposure differentially increases dose requirements of muscle relaxants. Therefore, careful monitoring of the patient is clinically essential to help reduce potential adverse events.

In summary, the present study demonstrated that chronic Dex administration induces different magnitudes of resistance to NDMRs in rat diaphragm, which is related to increased immature $\mathrm{nAChR}$ subunit expressions. 


\section{ACKNOWLEDGMENTS}

Research supported by the National Natural Science Foundation of China (\#81171845). We thank Professor Liang Zhu (Department of Pharmacology, School of Medicine, Shanghai JiaoTong University) for technical assistance and strong support.

\section{REFERENCES}

Arts WF and Oosterhuis HJ (1977). Long-term effect of glucocorticosteroids on neuromuscular blocking in mice. $J$. Neurol. Neurosurg. Psychiatr. 40: 675-677.

Braun S, Askanas V, Engel WK and Ibrahim EN (1993). Long-term treatment with glucocorticoids increases synthesis and stability of junctional acetylcholine receptors on innervated cultured human muscle. J. Neurochem. 60: 1929-1935.

Chen D, Yang MR, Huang LN, Qiu YW, et al. (2014). Dexamethasoneinduced hyposensitivity to rocuronium in rat diaphragm associated with muscle fiber transformation. Mol. Med. Rep. 9: 527-534.

Fagerlund MJ and Eriksson LI (2009). Current concepts in neuromuscular transmission. Br. J. Anaesth 103: 108-114.

Garcia JG and Ma SF (2005). Polymerase chain reaction: a landmark in the history of gene technology. Crit. Care Med. 33 (Suppl 12): S429-S432.

Jeevendra Martyn JA, Fukushima Y, Chon JY, Yang HS, et al. (2006). Muscle relaxants in burns, trauma, and critical illness. Int. Anesthesiol. Clin. 44: 123-143.

Kaplan I, Blakely BT, Pavlath GK, Travis M, et al. (1990). Steroids induce acetylcholine receptors on cultured human muscle: implications for myasthenia gravis. Proc. Natl. Acad. Sci. U. S. A. 87: 8100-8104.

Leeuwin RS, Veldsema-Currie RD, van Wilgenburg H and Ottenhof M (1981). Effects of corticosteroids on neuromuscular blocking actions of d-tubocurarine. Eur. J. Pharmacol. 69: 165-173.

Ma K, Mallidis C, Bhasin S, Mahabadi V, et al. (2003). Glucocorticoid-induced skeletal muscle atrophy is associated with upregulation of myostatin gene expression. Am. J. Physiol. Endocrinol. Metab. 285: E363-E371.

Maestrone E, Lagostena L, Henning RH, Hertog AD, et al. (1995) Functional aspects of dexamethasone upregulated nicotinic acetylcholine receptors in C2C12 myotubes. Pharmacol. Res. 32: 305-308.

Martyn JA and Richtsfeld M (2006). Succinylcholine-induced hyperkalemia in acquired pathologic states: etiologic factors and molecular mechanisms. Anesthesiology 104: 158-169.

Martyn JA, White DA, Gronert GA, Jaffe RS, et al. (1992). Up-and-down regulation of skeletal muscle acetylcholine receptors. Effects on neuromuscular blockers. Anesthesiology 76: 822-843.

Martyn JA, Fagerlund MJ and Eriksson LI (2009). Basic principles of neuromuscular transmission. Anaesthesia 64 (Suppl 1): $1-9$.

Parr SM, Galletly DC and Robinson BJ (1991). Betamethasone-induced resistance to vecuronium: a potential problem in neurosurgery? Anaesth. Intensive Care 19: 103-105.

Robinson BJ, Lee E, Rees D, Purdie GL, et al. (1992). Betamethasone-induced resistance to neuromuscular blockade: a comparison of atracurium and vecuronium in vitro. Anesth Analg. 74: 762-765.

Shima H (1990). The effect of corticosteroids on the recovery from vecuronium induced block. Masui 39: 619-625.

Shin YS, Fink H, Khiroya R, Ibebunjo C, et al. (2000). Prednisolone-induced muscle dysfunction is caused more by atrophy than by altered acetylcholine receptor expression. Anesth Analg. 91: 322-328.

Soltész S, Mencke T, Mey C, Röhrig S, et al. (2008). Influence of a continuous prednisolone medication on the time course of neuromuscular block of atracurium in patients with chronic inflammatory bowel disease. Br. J. Anaesth 100: 798-802.

Soltész S, Mencke T, Stunz M, Diefenbach C, et al. (2009). Attenuation of a rocuronium-induced neuromuscular block in patients receiving prednisolone. Acta Anaesthesiol. Scand. 53: 443-448.

Van Balkom RH, Zhan WZ, Prakash YS, Dekhuijzen PN, et al. (1997). Corticosteroid effects on isotonic contractile properties of rat diaphragm muscle. J. Appl. Physiol. 83: 1062-1067.

Wang H, Yang B, Xu YF, Yan T, et al. (2010). Different magnitude of resistance to nondepolarizing muscle relaxants in the denervated mouse skeletal muscle. Acta Pharmacol. Sin. 31: 399-404.

Yamamoto D, Maki T, Herningtyas EH, Ikeshita N, et al. (2010). Branched-chain amino acids protect against dexamethasone-induced soleus muscle atrophy in rats. Muscle Nerve 41: 819-827. 